\title{
Density fluctuations measured by ISEE 1-2 in the Earth's magnetosheath and the resultant scattering of radio waves
}

\author{
C. Lacombe, J.-L. Steinberg, C. C. Harvey, D. Hubert, A. Mangeney, M. Moncuquet \\ URA 264 du CNRS, DESPA, Observatoire de Paris, 92195 Meudon, France
}

Received: 20 June 1996 / Revised: 4 November 1996 / Accepted: 21 November 1996

\begin{abstract}
Radio waves undergo angular scattering when they propagate through a plasma with fluctuating density. We show how the angular scattering coefficient can be calculated as a function of the frequency spectrum of the local density fluctuations. In the Earth's magnetosheath, the ISEE 1-2 propagation experiment measured the spectral power of the density fluctuations for periods in the range 300 to $1 \mathrm{~s}$, which produce most of the scattering. The resultant local angular scattering coefficient can then be calculated for the first time with realistic density fluctuation spectra, which are neither Gaussian nor power laws. We present results on the variation of the local angular scattering coefficient during two crossings of the dayside magnetosheath, from the quasi-perpendicular bow shock to the magnetopause. For a radio wave at twice the local electron plasma frequency, the scattering coefficient in the major part of the magnetosheath is $b\left(2 f_{p}\right) \simeq 0.5-4 \times 10^{-9}$ $\operatorname{rad}^{2} / \mathrm{m}$. The scattering coefficient is about ten times stronger in a thin sheet $\left(0.1\right.$ to $\left.1 R_{E}\right)$ just downstream of the shock ramp, and close to the magnetopause.
\end{abstract}

\section{Introduction}

Radio waves are strongly scattered by density fluctuations when they propagate at frequencies from one to four times the local electron plasma frequency $f_{p}$. This scattering is due to fluctuations of the refractive index, and it changes the angular size, the direction, the intensity and the time-profile of radio emissions (Steinberg et al., 1971). The intrinsic size and direction of lowfrequency radio sources observed in the interplanetary medium cannot be determined without an estimation of the interplanetary scattering coefficient. Scattering has been taken into account in studies of the terrestrial auroral kilometric radiation AKR (Alexander et al., 1979; Steinberg et al., 1990), of the interplanetary Type-
III solar radio bursts (Steinberg et al., 1985), of the $2 f_{p}$ source upstream of the Earth's bow shock (Lacombe et al., 1988$)$ and of the outer heliospheric source at 2-3 $\mathrm{kHz}$ (Cairns, 1995). The angular size of the $2 f_{p}$ source is consistent with an angular scattering coefficient $b\left(2 f_{p}\right)=2$ to $4 \times 10^{-10} \mathrm{rad}^{2} / \mathrm{m}$ in the solar wind at 1 AU (Lacombe et al., 1988).

In situ measurements in the solar wind at $1 \mathrm{AU}$ have shown that the relative density fluctuations $\sigma_{N} / N$ are more intense downstream of interplanetary shocks (Huddleston et al., 1995; see also Woo, 1988); $\sigma_{N}$ is the standard deviation of the density $N$, for fluctuations with periods in the range $10 \mathrm{~min}$ to $1 \mathrm{~h}$. The average value of $\sigma_{N} / N$ is $15 \%$ between the interplanetary shock and the driver gas, but it is smaller than $6 \%$ in the interstream regions. Such a relative increase in the density fluctuations can also occur in the Earth's magnetosheath, downstream of the bow shock. However, the efficiency of the radio wave scattering does not only depend on $\sigma_{N} / N$, but also on the scale length $a$ of the fluctuations: high-frequency (small-scale) fluctuations produce a stronger scattering. The scale $a$, as well as $\sigma_{N} / N$, may change downstream of the bow shock.

The properties of the terrestrial AKR sources were measured from ISEE 3 in the dawn solar wind around 03:00 LT (Steinberg et al., 1989). From this position, the AKR source was observed through the flanks of the magnetosheath, a region for which $X_{G S E}<0 . X_{G S E}$ is the distance along the Earth-Sun line, positive towards the Sun. The angular radius of the source was deduced from the spin modulation of the signal received by a dipole much shorter than the wavelength. The source was assumed to be circular and uniformly bright. Using a Monte-Carlo ray-tracing method, Steinberg and Hoang (1993) calculated the refracted and scattered image of AKR sources, using two different values of the angular scattering coefficient $b\left(2 f_{p}\right)=1.6 \times 10^{-9} \mathrm{rad}^{2} / \mathrm{m}$ and $b\left(2 f_{p}\right)=4 \times 10^{-9} \mathrm{rad}^{2} / \mathrm{m}$ at twice the local $f_{p}$, in the flanks of the magnetosheath. They deduced the angular radius of the equivalent source (circular and uniformly bright). They showed that the direction and the size of 
the apparent AKR source recorded on board ISEE 3, on three different days, can be explained by the propagation and scattering of the AKR waves through the flanks of the magnetosheath: with the density distribution model of that region derived from in situ observations (Steinberg and Lacombe, 1992), a good fit to the observations is obtained with $b\left(2 f_{p}\right)=4 \times 10^{-9} \mathrm{rad}^{2} / \mathrm{m}$, in the flanks of the magnetosheath; while the other value tried, $b\left(2 f_{p}\right)=1.6 \times 10^{-9} \mathrm{rad}^{2} / \mathrm{m}$, is too small to account for the observations. In the calculations of Steinberg and Hoang, $b\left(2 f_{p}\right)$ is assumed to be constant from the bow shock to the magnetopause. This assumption was made to limit the number of parameters in the model of Steinberg and Hoang. We want to check the validity of this assumption.

There are no in situ measurements of the small-scale density fluctuations across the dawn flank of the Earth's magnetosheath. We are thus not able to estimate the local radio wave angular scattering in this region and to check whether the scattering coefficient is more intense in regions close to the bow shock and the magnetopause than in the middle of the magnetosheath at 03:00 LT. However, ISEE 1-2 has performed some complete crossings of the dayside $\left(X_{G S E}>0\right)$ magnetosheath, during which high time-resolution values of the electron density were acquired. We shall thus use the spectral power of the ISEE 1-2 density fluctuations, up to $1 \mathrm{~Hz}$, to estimate the scattering coefficient $b\left(2 f_{p}\right)$ at different depths in the dayside magnetosheath. We shall compare these local estimations with the overall estimation obtained by Steinberg and Hoang for the dawn flank of the magnetosheath.

\section{The scattering coefficient of radio waves}

A radio wave with a frequency $f$ undergoes an angular scattering due to local random fluctuations of the refractive index $\mu$. When the magnetic field is neglected

$\mu^{2}=1-f_{p}^{2} / f^{2}$.

The index fluctuations are related to the density fluctuations by

$\delta \mu^{2}=\frac{1}{4 \mu^{2}} \frac{f_{p}^{4}}{f^{4}} \frac{\delta N^{2}}{N^{2}}$.

The total mean square fluctuation of the refractive index can be expressed in terms of the power density in wave number space, thus

$<\delta \mu^{2}>=4 \pi \int_{0}^{\infty} P_{\mu}(q) q^{2} d q$,

where $P_{\mu}(q)$ is the local 3D power spectrum of the refractive index fluctuations for a wave vector $\boldsymbol{q}$. We assume that both the density fluctutations and the wave number spectrum are isotropic.

The mean square angular deviation per unit path length along a ray is $b(f)=\frac{2 I}{\mu^{2}} \operatorname{rad}^{2} / \mathrm{m}$,

where (Hollweg, 1970, Eq. 23)

$I=2 \pi^{2} \int_{0}^{\infty} P_{\mu}(q) q^{3} d q$.

The factor 2 in Eq. 3 implies that the non-correlated angular deviations in two orthogonal planes containing the ray are taken into account. The spectral power $P_{\mu}(q)$ of the refractive index is related to the spectral power of the density fluctuations by

$P_{\mu}(q)=\frac{1}{4 \mu^{2} N^{2}} \frac{f_{p}^{4}}{f^{4}} P_{N}(q)$.

Equations 3, 4 and 5 give

$b(f)=\frac{\pi^{2}}{\mu^{4} N^{2}} \frac{f_{p}^{4}}{f^{4}} \int_{0}^{\infty} P_{N}(q) q^{3} d q \operatorname{rad}^{2} / \mathrm{m}$.

The electron density $N$ is measured locally as a function of time. To relate the frequency $v$ of the observed density fluctuations to the wave vector $\boldsymbol{q}$, we assume that the phase velocity of the fluctuations is zero in the plasma frame, which moves with a velocity $\boldsymbol{V}$ with respect to the spacecraft; then

$2 \pi v=\boldsymbol{q} \cdot \boldsymbol{V}$,

in which $\boldsymbol{q} . \boldsymbol{V}$ and $v$ can be positive or negative. In Sect. 5.1 we shall discuss the validity of assumption (7) in the magnetosheath.

The measured spectral power of the density fluctuations is

$$
\begin{aligned}
P_{N}(v) & =\int_{-\infty}^{\infty} \int_{-\infty}^{\infty} \int_{-\infty}^{\infty} P_{N}(q) \delta\left(v-\frac{\boldsymbol{q} \cdot \boldsymbol{V}}{2 \pi}\right) d^{3} q \\
& =\frac{4 \pi^{2}}{V} \int_{0}^{\infty} P_{N}(q) q d q,
\end{aligned}
$$

so that all the wave vectors $q$ contribute to $P_{N}(v)$.

The integral of Eq. 6 can be calculated as a function of the measured spectrum $P_{N}(v)$ for positive values of $v$ :

$$
\begin{aligned}
\int_{0}^{\infty} P_{N}(v) v d v & =\int_{0}^{\infty} v d v \int_{-\infty}^{\infty} \int_{-\infty}^{\infty} \int_{-\infty}^{\infty} P_{N}(q) \delta\left(v-\frac{\boldsymbol{q} \cdot \boldsymbol{V}}{2 \pi}\right) d^{3} q \\
& =\frac{V}{2} \int_{0}^{\infty} P_{N}(q) q^{3} d q .
\end{aligned}
$$

The scattering coefficient can then be expressed as a function of the spectrum of the density fluctuations for positive frequencies

$b(f)=\frac{2 \pi^{2}}{\mu^{4}} \frac{f_{p}^{4}}{f^{4}} \frac{1}{N^{2} V} \int_{0}^{\infty} P_{N}(v) v d v \operatorname{rad}^{2} / \mathrm{m}$,

where $V$ is in $\mathrm{m} / \mathrm{s}, N$ in $\mathrm{cm}^{-3}, v$ in $\mathrm{Hz}$ and $P_{N}$ in $\mathrm{cm}^{-6}$ $\mathrm{Hz}^{-1}$. This local formula is only valid for small scattering angles and a constant mean value of $\mu$. It also implies that the radio wavelength $\lambda=c / \mu f$ is much smaller than the average scale $(\simeq V / 2 v)$ of the density fluctuations (Steinberg et al., 1971). 
For an observed Gaussian distribution of density fluctuations, $P_{o b s}(q)=P_{0} \exp \left(-q^{2} a^{2}\right)$, the total scattering coefficient in two orthogonal planes, calculated using Eq. 6, is

$b(f)=\frac{\pi^{1 / 2}}{\mu^{4}} \frac{f_{p}^{4}}{f^{4}} \frac{1}{a}\left[\frac{\Delta N}{N}\right]^{2}$,

where

$[\Delta N]^{2}=\int_{0}^{\infty} P_{o b s}(q) d q=\frac{4 \pi}{2} \int_{0}^{\infty} P_{N}(q) q^{2} d q$.

We have checked that this scattering coefficient (Eq. 11) is equal to the value found by Steinberg et al. (1971) for a Gaussian autocorrelation function of the refractive index fluctuations. If the density fluctuation spectrum obeys a power law $P_{N}(v)=P_{0}\left(v / v_{\text {min }}\right)^{-\alpha}$ between $v_{\text {min }}$ and $v_{\max }$, and $P_{N}(v)=P_{0}$ between 0 and $v_{\min }$, we obtain

$b(f)=\frac{2 \pi^{2}}{\mu_{4}} \frac{f_{p}^{4}}{f^{4}} \frac{P_{0} v_{\min }^{2}}{N^{2} V}\left[\frac{1}{2}+\frac{1}{\alpha-2}\left[1-\left(\frac{v_{\min }}{v_{\max }}\right)^{\alpha-2}\right]\right]$,

where the term $1 / 2$ corresponds to the scattering by fluctuations between 0 and $v_{\min }$. [Equation 12 of Lacombe et al. (1988) is only an approximate formula for $\alpha<2,2 \pi v=q V$ and $v_{\min } \ll v_{\max }$, in which the fluctuation spectrum takes into account the negative and positive frequencies $v$.]

To allow comparisons between different media, it is convenient to eliminate the first part of the expression for $b(f)$ which is a function of $f_{p} / f$, so that the scattering coefficient of radio waves is generally given at $2 f_{p}$ (Steinberg and Hoang, 1993). If we take into account the boundaries of the frequency domain, $v_{\min }$ and $v_{\max }$ in the spacecraft frame, Eq. 10 becomes

$b\left(2 f_{p}\right)=\frac{2 \pi^{2}}{9} \frac{1}{N^{2} V} \int_{v_{\min }}^{v_{\max }} P_{N}(v) v d v \operatorname{rad}^{2} / \mathrm{m}$.

In the Earth's magnetosheath, Alexander et al. (1979) assumed that the density fluctuation spectrum was Gaussian with $a=1000 \mathrm{~km}$ and $\Delta N / N=50 \%$ : Eq. 11 then gives $b\left(2 f_{p}\right)=50 \times 10^{-9} \mathrm{rad}^{2} / \mathrm{m}$. Steinberg and Hoang (1993) found that the size and the direction of some AKR sources seen through the dawn magnetosheath flank, on three different days, implied weaker scattering coefficients: a good fit to the observations was obtained with $b\left(2 f_{p}\right) \simeq 4 \times 10^{-9} \mathrm{rad}^{2} / \mathrm{m}$, while the value $b\left(2 f_{p}\right)=1.6 \times 10^{-9} \mathrm{rad}^{2} / \mathrm{m}$ was too small. We emphasize that the ray tracing does not rely on an assumed Gaussian or power law shape of the density fluctuations, but only on the value of $b\left(2 f_{p}\right)$, which is an integral of the inverse of the scale length of the density fluctuations over the spectrum (Eq. 14).

In Sect. 3, we consider the values of $b\left(2 f_{p}\right)$ deduced from the electron density measured on ISEE 1-2 with a time resolution of about $1 \mathrm{~s}$.

\section{ISEE 1-2 data}

In the following, we shall assume that $V=100 \mathrm{~km} / \mathrm{s}$ in the magnetosheath. Since, with ISEE 1-2, we cannot precisely measure density fluctuations on frequencies larger than $v_{\max } \simeq 1 \mathrm{~Hz}$, the smallest scale length we can reach (inner scale) is $l_{i} \simeq V /\left(2 v_{\max }\right)=50 \mathrm{~km}$ : the basic assumption for scattering calculations is fulfilled because $l_{i}$ is larger than $\lambda \simeq 10 \mathrm{~km}$, the wavelength corresponding to the lowest frequency $(30 \mathrm{kHz})$ recorded on board ISEE 3. On the three days considered, in the magnetosheath, the ion skin depth (which is a minimum scale for plasma waves below the proton gyrofrequency) was $c / \omega_{p i} \simeq 25-50 \mathrm{~km}$, i.e. of the same order of magnitude as the inner scale reached by the ISEE 1-2 density data. Each spectrum $P_{N}(v)$ will be calculated with a fast Fourier transform in a sliding window with 5 $\min 20 \mathrm{~s}$ of data (1280 points). The spectral power of the density fluctuations will be calculated down to $v_{\min }=0.0156 \mathrm{~Hz}$, which implies an outer scale $l_{o} \simeq V /\left(2 v_{\min }\right)=3200 \mathrm{~km}$, i.e. half the Earth's radius.

The first magnetosheath crossing analysed in this paper (Figs. 1 and 2) occurred on day 247/80/09/03 [already analysed by Moustaizis et al. (1986), Hubert (1994) and Lacombe et al. (1992)]. The propagation experiment (Harvey et al., 1978) gives the average density between ISEE 1 and ISEE 2 . The separation $S$ between ISEE 1 and ISEE 2 was 50 to $75 \mathrm{~km}$, i.e. slightly larger than the considered inner scale $l_{i} \simeq 50 \mathrm{~km}$. This implies an underestimation of the spectral power $P_{N}(q)$ by a factor
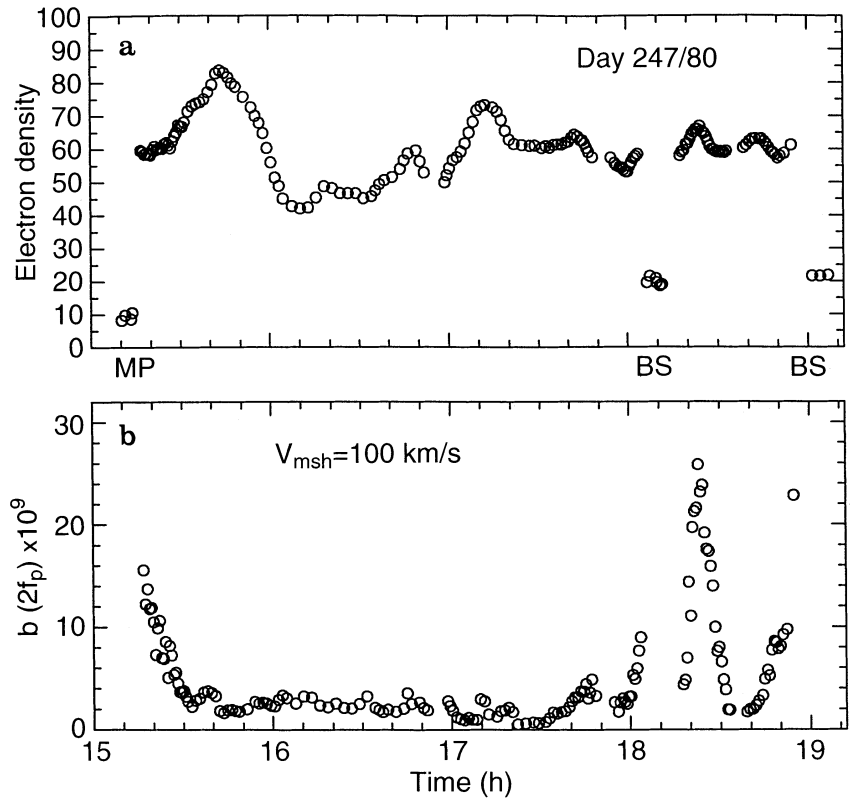

Fig. 1.a The electron density in the magnetosheath $\left(\right.$ in $\mathrm{cm}^{-3}$, averaged in a sliding window of $5 \mathrm{~min} 20 \mathrm{~s}$ ) from the magnetopause MP to the bow shock BS (day 247/80). b The scattering coefficient of radio waves $b\left(2 f_{p}\right)$ (in $10^{-9} \mathrm{rad}^{2} / \mathrm{m}$ ) calculated (Eq. 14) between $v_{\min }=0.0156 \mathrm{~Hz}$ and $v_{\max }=1 \mathrm{~Hz}$, under the assumption that the flow velocity is $V_{m s h}=100 \mathrm{~km} / \mathrm{s}$ in the magnetosheath 

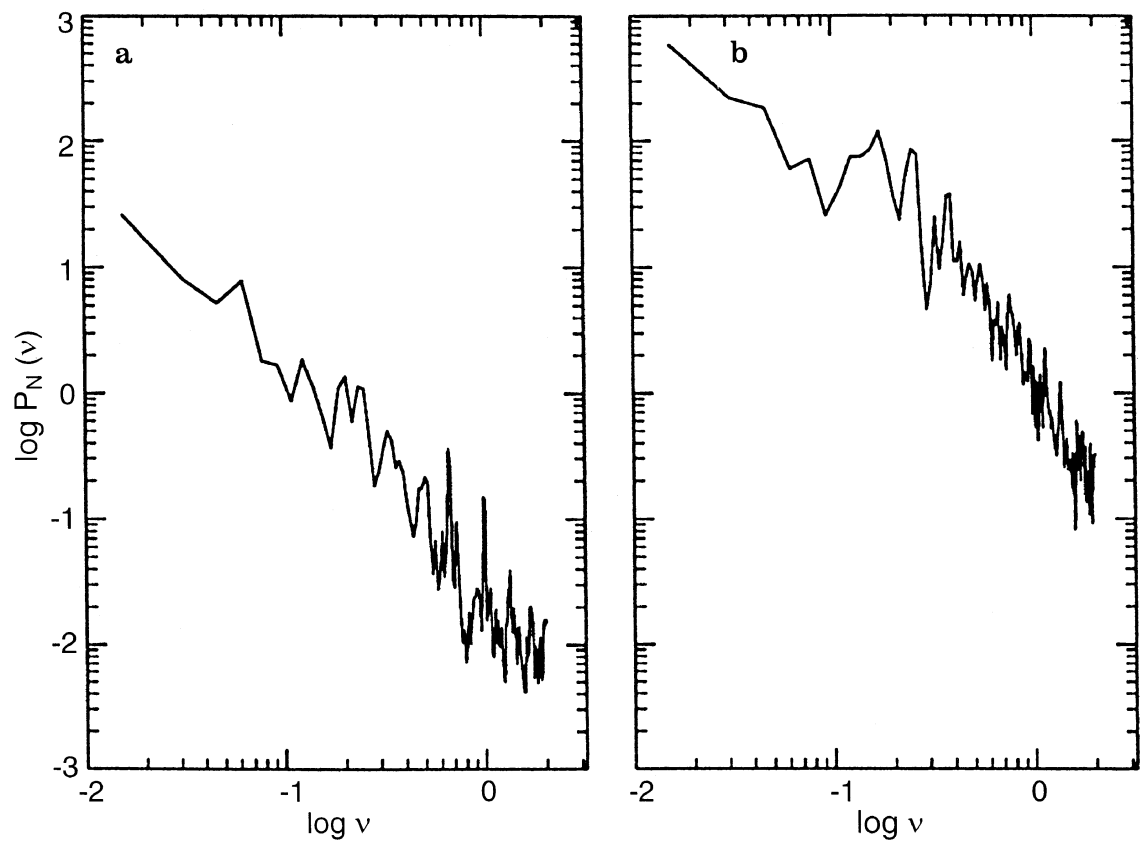

Fig. 2a, b. Spectra $\left(\right.$ in $\left.\mathrm{cm}^{-6} \mathrm{~Hz}^{-1}\right)$ of the electron density measured over $5 \mathrm{~min} 20 \mathrm{~s}$ by ISEE 1-2 in the magnetosheath, day 247/80. a At 17:22 UT, in the middle of the magnetosheath. The peaks at 0.66 and $0.99 \mathrm{~Hz}$ are artefacts; they have been removed for the calculation of the scattering coefficient $b\left(2 f_{p}\right)$; b at 18:23 UT, close to the bow shock $\frac{\sin ^{2}(\mathbf{q} \cdot \mathbf{S} / 2)}{(\mathbf{q} \cdot \mathbf{S} / 2)^{2}}$

for fluctuations with a wavelength smaller than $S$ (Celnikier et al. 1983; Hubert et al., manuscript in preparation, 1997). However, the density data are sometimes noisy at the highest frequencies, so that $P_{N}(v)$ can be overestimated close to the Nyquist frequency, $2 \mathrm{~Hz}$. We have not used the methods presented by Celnikier et al. $(1983,1987)$ to "purify" the density data. But we have withdrawn possible spectral artefacts, namely peaks at multiples of the spacecraft spin frequency around $0.33,0.66$ and $0.99 \mathrm{~Hz}$. Then, $b\left(2 f_{p}\right)$ has been numerically calculated for each spectrum with Eq. 14, as well as the standard deviation of the density

$\Delta N=\left[\int_{v_{\min }}^{v_{\max }} P_{N}(v) d v\right]^{1 / 2}$.

Another complete magnetosheath crossing occurred on day 242/81/08/30 (Figs. 3 and 4). A part of the highfrequency noise of the density data of that day has been removed using an improved Savitzky-Golay method (Press et al., 1992, p. 644). The separation between ISEE 1 and 2 was between 160 and $240 \mathrm{~km}$, so that some highfrequency density fluctuations are smoothed out by the propagation experiment (Eq. 15). As the highest frequencies (smallest scales) produce the strongest scattering, the scattering coefficient calculated by Eq. 14 with probably be underestimated.

On day 188/81/07/07 (Figs. 5 and 6), the separation was $54 \mathrm{~km}$. The density fluctuations were only measured close to the bow shock, on the dusk flank of the magnetosheath.

A different method of evaluating the scattering coefficient has been tried. We have approximated a measured density spectrum by one or two power laws in different frequency ranges, and then deduced the "fitted" $b\left(2 f_{p}\right)$ with Eq. 13. This fitting has been made for several spectra on the three days considered. In the solar wind, the spectral indices are relatively small: 1.7 below $0.1 \mathrm{~Hz}$, and less than 1 above $0.1 \mathrm{~Hz}$ (Celnikier et $a l ., 1987)$. In the magnetosheath we find spectral indices smaller than 2 at low frequencies, but larger than 2 at higher frequencies. Even when the fit of the power laws to the data is relatively good, the ratio between the "fitted" $b\left(2 f_{p}\right)$ and the calculated $b\left(2 f_{p}\right)$ can reach 3 to 4. It is thus more precise to calculate $b\left(2 f_{p}\right)$ by numerical
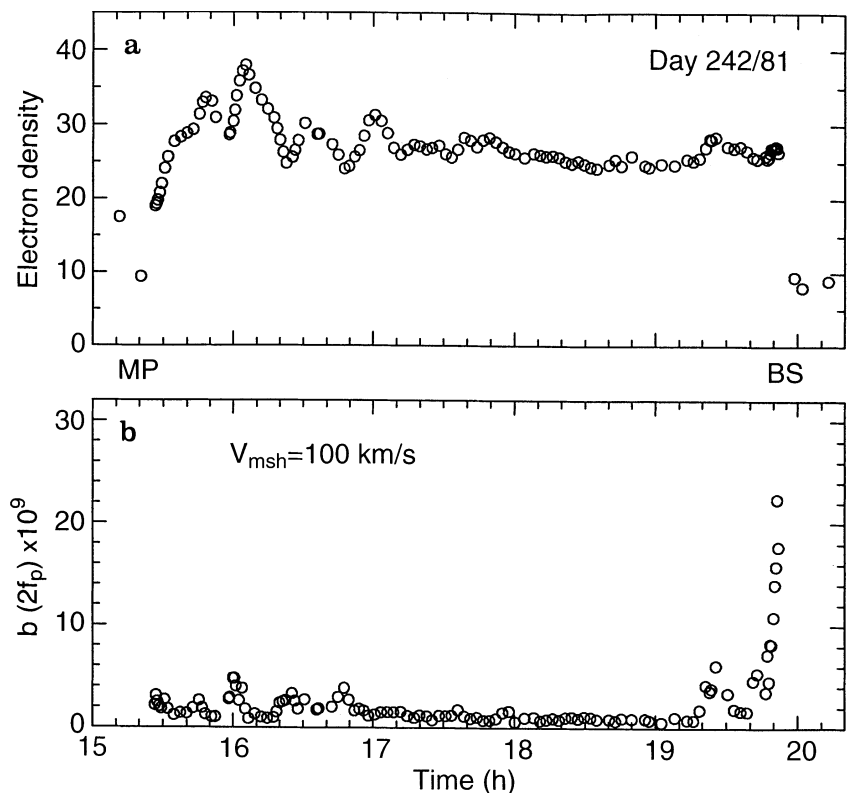

Fig. 3.a The electron density in the magnetosheath from the magnetopause boundary layer MP to the bow shock BS (day 242/ 81 ). b The scattering coefficient of radio waves $b\left(2 f_{p}\right)$ (same as Fig. 1; $V_{m s h}=100 \mathrm{~km} / \mathrm{s}$ ) 

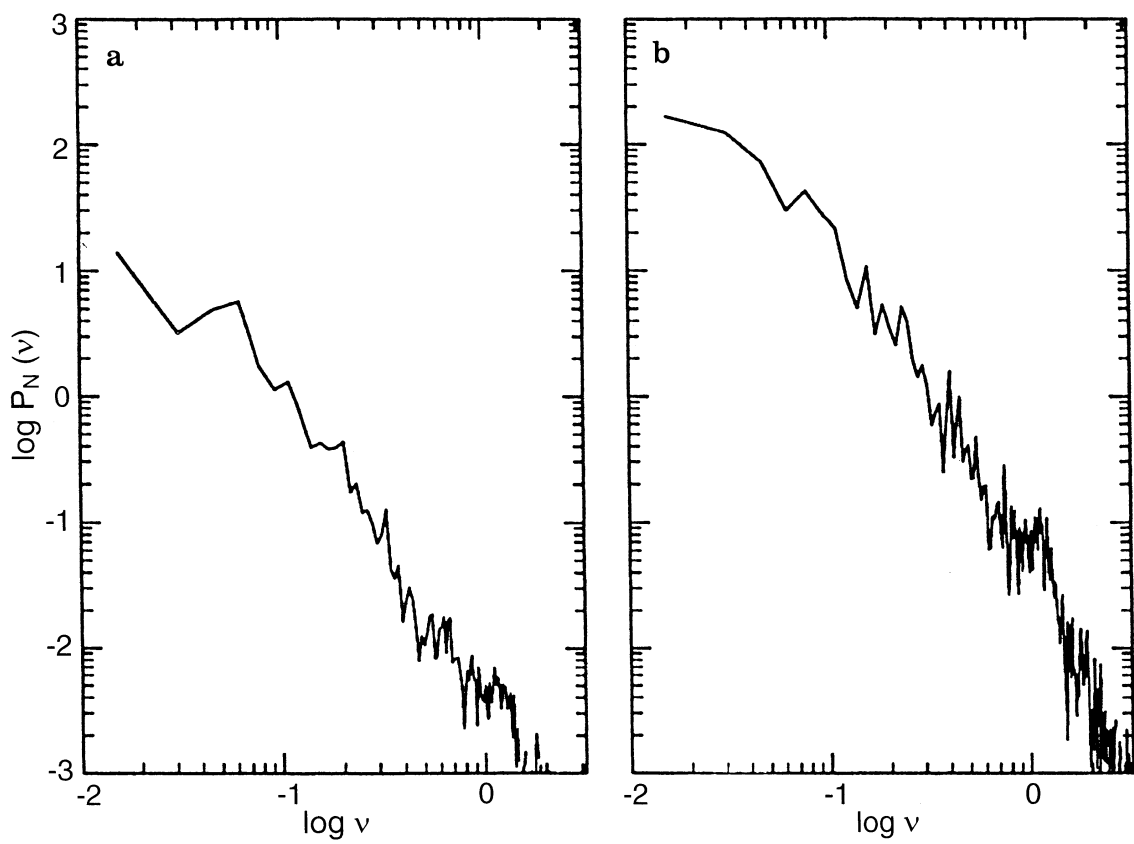

Fig. 4a, b. Spectra $\left(\right.$ in $\mathrm{cm}^{-6} \mathrm{~Hz}^{-1}$ ) of the electron density measured over 5 min $20 \mathrm{~s}$ by ISEE $1-2$ in the magnetosheath, day $242 / 81$. a At 17:31 UT, in the middle of the magnetosheath; b at 19:52 UT close to the bow shock integration, inasmuch as the fit with power laws is impossible for some spectra: see for instance Figs. $2 b$ and $6 a$.

Table 1 contains the characteristics of the considered shock crossings, calculated with parameters given by Couzens and King (1986): the total upstream $\beta_{u}$ factor, the Alfvén Mach number $M_{A N}$ along the shock normal, and the angle $\theta_{B N}$ between the upstream magnetic field and the shock normal. $S$ is the satellite separation, and $c / \omega_{\text {pid }}$ the downstream ion skin depth or inertial length. The downstream $\beta$ is certainly larger than 1 during the considered magnetosheath intervals.
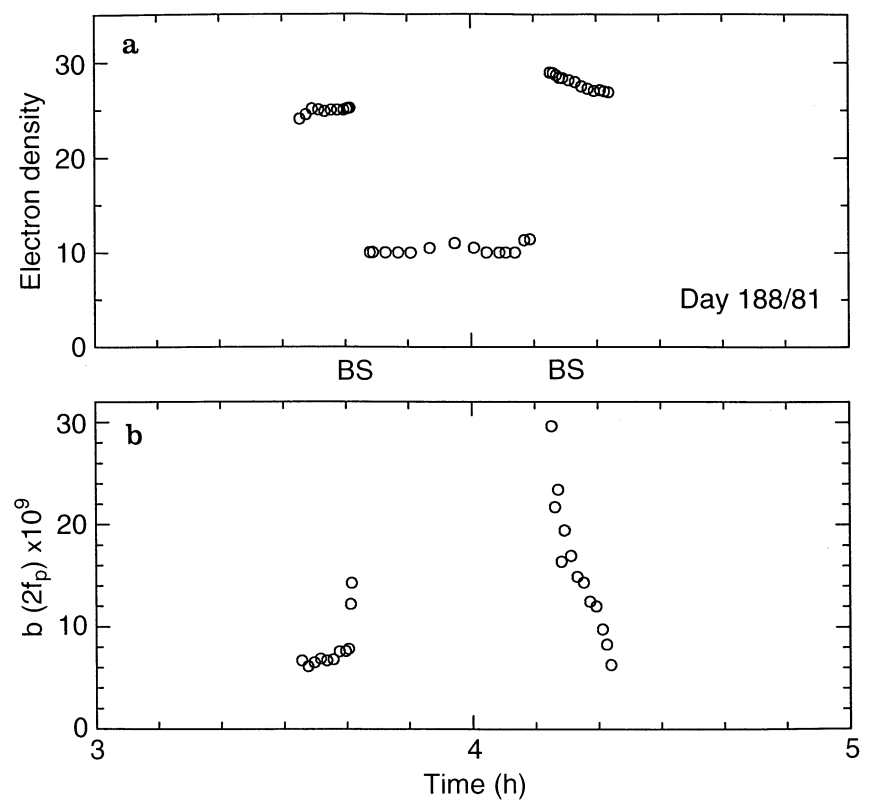

Fig. 5a The electron density close to the bow shock BS (day 188/81). b The scattering coefficient of radio waves $b\left(2 f_{p}\right)$ (same as Fig. 1; $V_{m s h}=100 \mathrm{~km} / \mathrm{s}$ )

\section{Results}

\subsection{Day $247 / 80$}

Figure 1 shows the average density $N$ and the scattering coefficient $b\left(2 f_{p}\right)$ observed from the magnetopause MP to the bow shock crossings $\mathrm{BS} ; b\left(2 f_{p}\right)$ has been calculated neither in the magnetosphere nor in the solar wind. The magnetopause crossing (15:15 UT) occurred at $X=7.4 R_{E}, Y=-0.9 R_{E}$ and $Z=2.4 R_{E}$ in GSE coordinates $\left(Z_{G S E}\right.$ is perpendicular to the ecliptic plane, positive northward); the last shock crossing (18:58 UT), at $X=12.3 R_{E}, Y=1.6 R_{E}$ and $Z=2.5 R_{E}$. The two first shock crossings occurred at 18:07 and 18:14, with a partial reentry in the solar wind at 18:21 (Lacombe et al., 1992). For each $5 \mathrm{~min} 20 \mathrm{~s}$ interval, we have calculated the average density $N$ and the standard deviation (Eq. 16): the ratio $\Delta N / N$ is always smaller than $10 \%$

Figure 2 shows two spectra of the density fluctuations. The spectrum 2a, with a low power level, was measured at 17:22 UT, in the middle of the sheath; the high-power spectrum, at 18:23 UT, was measured close to a shock crossing. More than 150 similar spectra were calculated during the same magnetosheath crossing, with overlapping time intervals.

In the major part of the magnetosheath, from 15:30 to $17: 40 \mathrm{UT}$ (Fig. 1), $b\left(2 f_{p}\right)$ varies between 0.5 and $410^{-9} \mathrm{rad}^{2} / \mathrm{m}$; the average value is $b\left(2 f_{p}\right)=2.2$ $\pm 0.810^{-9} \mathrm{rad}^{2} / \mathrm{m}$. But $b\left(2 f_{p}\right)$ reaches $1610^{-9} \mathrm{rad}^{2} / \mathrm{m}$ close to the magnetopause and 4 to $2610^{-9} \mathrm{rad}^{2} / \mathrm{m}$ close to the bow shock crossings. Close to these discontinuities, enhanced values of $b\left(2 f_{p}\right)$ are seen for about 10 min, which correspond to thicknesses of 1300 to 2000 $\mathrm{km}$ if we take into account the spacecraft velocity, $v_{s / c}=2.2$ to $3.4 \mathrm{~km} / \mathrm{s}$. There is thus a gradient of $b\left(2 f_{p}\right)$ close to strong discontinuities: $b\left(2 f_{p}\right)$ is multiplied by 10 over roughly $1700 \mathrm{~km}$. 

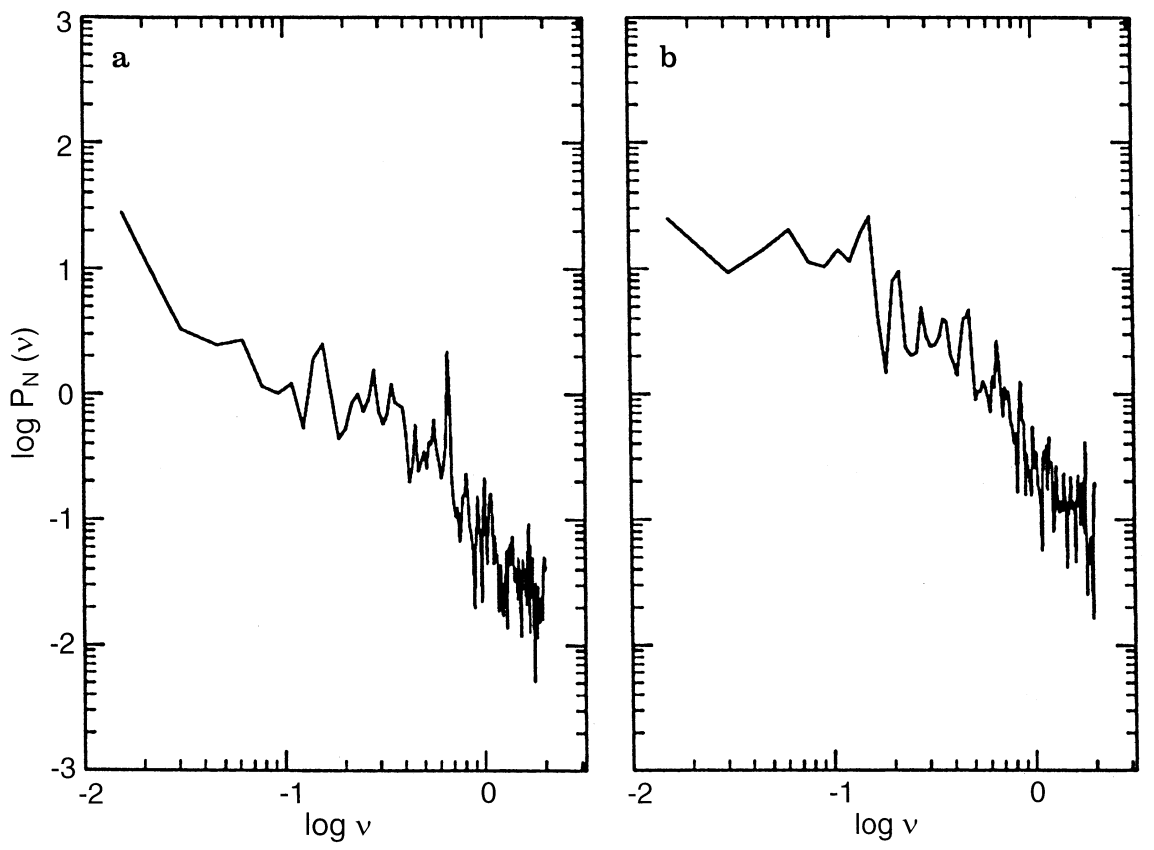

Fig. 6a,b. Spectra (in $\mathrm{cm}^{-6} \mathrm{~Hz}^{-1}$ ) of the electron density measured during 5 min $20 \mathrm{~s}$ by ISEE $1-2$ in the magnetosheath, day $188 / 81$. a At 03:34 UT, relatively far from the shock. The peaks at 0.66 and $0.99 \mathrm{~Hz}$ are artefacts. They have been removed for the calculation of the scattering coefficient $b\left(2 f_{p}\right)$; b at 04:13 UT, close to the bow shock

Table 1. Parameters for the bow shock crossings on ISEE 1-2

\begin{tabular}{lllllll}
\hline Date & UT & $S(\mathrm{~km})$ & $\beta_{\mu}$ & $\theta_{B N}$ & $M_{A N}$ & $c / \omega_{\text {pid }}$ \\
\hline $247 / 80$ & $18: 07$ & 55 & 1.7 & 52 & 7.1 & $30 \mathrm{~km}$ \\
$247 / 80$ & $18: 14$ & 54 & 1.3 & 44 & 6.4 & $30 \mathrm{~km}$ \\
$247 / 80$ & $18: 58$ & 51 & 2.9 & 75 & 6.5 & $29 \mathrm{~km}$ \\
$188 / 81$ & $03: 44$ & 55 & 1.7 & 88 & 8.8 & $48 \mathrm{~km}$ \\
$188 / 81$ & $04: 10$ & 54 & 1.6 & 78 & 9.0 & $42 \mathrm{~km}$ \\
$242 / 81$ & $19: 58$ & 161 & 1.3 & 45 & 8.7 & $44 \mathrm{~km}$ \\
$278 / 81$ & $17: 32$ & 480 & 2.0 & 15 & 8.0 & $72 \mathrm{~km}$ \\
\hline
\end{tabular}

Two weak discontinuities are also observed in the magnetosheath at 17:12 and 17:18 UT, and the resultant values of $b\left(2 f_{p}\right)$ are three times larger than in the surrounding sheath (Fig. 1). As noted by Huddleston et al. (1995), density fluctuations are good tracers of propagating disturbances.

As shown by Moustaizis et al. (1986), well-developed mirror modes with a period around $15 \mathrm{~s}$ were dominant near the magnetopause, from 15:30 to 16:00 UT, and Alfvénic waves were dominant closer to the shock, from 16:25 to 18:00 UT; the scattering coefficients are not significantly different in these two intervals (Fig. 1). However, the smallest values of the scattering coefficient are observed between 17:20 and 17:30 UT (Figs. 1 and 2a), an interval during which pure Alfvén ion cyclotron waves are observed, without any anticorrelation between the density and the magnetic field, i.e. without mirror mode at any frequency. Thus, Alfvén ion cyclotron waves, which are only weakly compressive, probably produce weaker scattering than mirror modes. The presence of Alfvénic waves in the upstream part of the dayside mangetosheath, and of mirror waves in the downstream part, has been observed during other magnetosheath crossings (Hubert et al., manuscript in preparation, 1997; see also Fairfield and Ness, 1970).

On that day (247/80), the subsolar point of the magnetopause was at $X=8.8 R_{E}$, i.e. about $1 R_{E}$ closer to the Earth than the fifth model of Table 2 of Sibeck et al. (1991): this corresponds to a highly compressed magnetosheath. Moreover, with the paraboloidal model of Filbert and Kellogg (1979), the subsolar points of the bow shock were at $X=11.5 R_{E}$ (at 18:07 UT) and $12.5 R_{E}$ (at 18:58), while the average value is about $15.1 R_{E}$ (Peredo et al., 1995). We have calculated $b\left(2 f_{p}\right)$ on another day (242/81) when the magnetosheath was less compressed.

\subsection{Day $242 / 81$}

Figure 3 shows the average magnetosheath density from the magnetopause MP (or the boundary layer) at 15:24 UT $\left(X=11.3 R_{E}, Y=2.1 R_{E}\right.$ and $\left.Z=0.0 R_{E}\right)$ to the bow shock crossing BS at 19:55 $\left(X=15.0 R_{E}\right.$, $Y=5.2 R_{E}$ and $\left.Z=-1.0 R_{E}\right)$. At the crossing times, the subsolar point of the magnetopause was at $X=$ $11.7 R_{E}$, close to the second model of Table 2 of Sibeck et al. (1991); and the subsolar point of the shock at $15.6 R_{E}$ : these values imply that the magnetosheath compression was close to its average value.

Figure 4 shows a density spectrum at 17:31 UT (middle of the sheath) and a spectrum at 19:52, close to the bow shock. The bit rate was four times higher than on day $247 / 80$, so that the Nyquist frequency was no longer $2 \mathrm{~Hz}$ but $8 \mathrm{~Hz}$. However, the scattering coefficient was still calculated for $v_{\max }=1 \mathrm{~Hz}$ : higher fre- 
quencies would correspond to scales smaller than the spacecraft separation, which cannot be correctly measured. The ratio $\Delta N / N$ is always smaller than $14 \%$.

Assuming that $V$ is still $100 \mathrm{~km} / \mathrm{s}$, we find once again that $b\left(2 f_{p}\right)$ is multiplied by 10 over $10 \mathrm{~min}$ close to the bow shock, that is over about $1000 \mathrm{~km}\left(v_{s / c}=1.5 \mathrm{~m} / \mathrm{s}\right)$. In the major part of the magnetosheath, from 15:25 to 19:20 UT, the average value is $b\left(2 f_{p}\right)=1.6 \pm 0.9$ $10^{-9} \mathrm{rad}^{2} / \mathrm{m}, 25 \%$ weaker than on day $247 / 80$. However, it is difficult to conclude that the scattering is stronger when the magnetosheath is highly compressed (day 247/ 80 ). Indeed the separation between ISEE 1 and 2, and the noise of the density data were different on the two days considered, so we cannot state that the difference between the scattering coefficients on these two days is significant (see Sect. 3).

We note in Fig. 3 that the scattering coefficient $b\left(2 f_{p}\right)$ does not increase close to the magnetopause at 15:24 UT. Actually, ISEE 1-2 was probably in the boundary layer from 15:15 to 15:24. Three other magnetopause crossings occurred between 14:45 and 14:51 UT, but the quality of the data was too poor to yield a valid spectrum of the density fluctuations.

\subsection{Day $188 / 81$}

On this day, ISEE 1-2 crossed the bow shock twice (Fig. 5) at $X=3.8 R_{E}, Y=19.6 R_{E}$ and $Z=-1.7 R_{E}$. The corresponding subsolar point of the shock was at $X=12.5 R_{E}$, implying a highly compressed magnetosheath. The relative standard deviation $\Delta N / N$ is always smaller than $9 \%$. Close to the bow shock, the increase of the scattering coefficient (Fig. 5) is smaller when ISEE 12 enters the solar wind, at 03:44 UT, than when ISEE 12 enters the magnetosheath, at 04:10. This difference may be due to the fact that the shock is more perpendicular at $03: 44\left(88^{\circ}\right.$, see Table 1) than at $04: 10$ $\left(78^{\circ}\right)$. But it is more probably due to slow rotations of the magnetic field which occurred at 03:33-03:34 and at 03:42-03:43 UT: such rotations could be related to back and forth motions of the shock, so that the distance of the spacecraft to the shock changed abruptly.

\section{Discussion}

\subsection{Uncertainties on the angular scattering coefficient}

The relation (Eq. 7) between the frequency and the wave vector of the density fluctuations relies on the assumption that the phase velocity of the density perturbations is negligible with respect to the flow velocity. Such an assumption is usually made in the solar wind, where the flow velocity is much larger than the Alfvén and sound velocities. But the flow velocity is clearly smaller in the magnetosheath. Two main wave modes are found in the dayside magnetosheath (Hubert, 1994), the mirror mode (with wave vectors at large angles of the ambient magnetic field) and the Alfvén ion cyclotron mode (Aic, with wave vectors mainly parallel to the field). The phase velocity of the mirror mode is indeed equal to zero. The phase velocity $v_{\varphi}$ of Aic waves is not zero, but such waves are found propagating downstream $\left(\boldsymbol{V}+\boldsymbol{v}_{\varphi}\right)$ as well as upstream $\left(\boldsymbol{V}-\boldsymbol{v}_{\varphi}\right)$ (Lacombe et al., 1995). It is thus roughly valid to neglect $v_{\varphi}$ with respect to $V$ for calculations of the scattering coefficient of radio waves in the magnetosheath.

Our results on the scattering coefficient of radio waves are functions of $v_{\min }$ and $v_{\max }$, i.e. the outer and the inner scales of the density fluctuations. For the six spectra shown in Figs. 2, 4 and 6, if $v_{\min }$ had been two times larger $(0.031 \mathrm{~Hz}), b\left(2 f_{p}\right)$ would have been reduced less than $10 \%$ : $v_{\min }$ is not a critical parameter. For the same six spectra, had we taken $v_{\max }$ equal to the Nyquist frequency $\left(2\right.$ or $8 \mathrm{~Hz}$ ) rather than $v_{\max }=1 \mathrm{~Hz}, b\left(2 f_{p}\right)$ would have been increased up to $45 \%$. And if $v_{\max }=0.3 \mathrm{~Hz}$ instead of $1 \mathrm{~Hz}, b\left(2 f_{p}\right)$ would have decreased $15 \%$ to $60 \%$ : $v_{\max }$ is a critical parameter because most of the scattering is due to small-scale inhomogeneities. The relative uncertainty on the spectral integral of Eq. 14 is thus $(\Delta S i) / S i=0.5$ to 1 . We have cut our spectra at $v_{\max }=1 \mathrm{~Hz}$ because there is a large uncertainty on $P_{N}(v)$ for $v>1 \mathrm{~Hz}$. Thanks to the steepness of the density spectra up to $1 \mathrm{~Hz}$, the frequency domain $(0.0156-1 \mathrm{~Hz})$ seems to be reasonably well suited for estimations of the order of magnitude of the scattering coefficient of radio waves in the magnetosheath. But we cannot exclude the existence of nonnegligible density fluctuations above 2 or $8 \mathrm{~Hz}$, which would produce an efficient scattering.

The scattering coefficient through the dayside $\left(X_{G S E}>0\right)$ magnetosheath has been estimated for a constant flow velocity $V=100 \mathrm{~km} / \mathrm{s}$ : according to Eq. $14, b\left(2 f_{p}\right)$ is proportional to $1 / V$. We have no measurement of $V$ in the magnetosheath during the three days considered. However, the solar wind velocity (Couzens and King, 1986) projected along the shock normal varies between 320 and $380 \mathrm{~km} / \mathrm{s}$ for the shocks listed in Table 1. With the observed shock density jump, the downstream normal velocity will be around 100 to $150 \mathrm{~km} / \mathrm{s}$, close to the shock. Far from the shock, it will be weaker. Indeed, the shape of the streamlines, and the modulus of the magnetosheath flow velocity are given by Figs. 9 and 10 of Spreiter and Stahara (1985) for a gas dynamic model: the flow velocity is larger close to the shock, and smaller close to the magnetopause, in the dayside magnetosheath. This trend could divide $b\left(2 f_{p}\right)$ by a factor 2 close to the bow shock. The relative uncertainty on the assumed flow velocity is $(\Delta V) / V=$ 0.5 to 1 .

We conclude that the relative uncertainty $(\Delta b) / b=(\Delta S i) / S i+(\Delta V) / V$ can be 1 to 2 for the local angular scattering coefficient of radio waves calculated at $2 f_{p}$ in the dayside magnetosheath.

In the flanks $\left(X_{G S E}<0\right)$ of the magnetosheath, the flow velocity can be three times larger than in the dayside magnetosheath (see Fig. 10 of Spreiter and Stahara). This velocity trend would result in a scattering coefficient three times smaller in the flanks than in the dayside magnetosheath, for a given spectrum of density fluctuations. Are the fluctuation spectra more intense in 
the flanks than in the dayside magnetosheath? There is no observation to answer this question, neither for the density nor for the magnetic field spectra. Where the magnetosheath waves are controlled by the bow shock properties, the fluctuations in the flanks will probably be weaker because the normal Mach number $M_{A N}$ is two or three times smaller on the flanks than close to the nose of the bow shock. Close to the magnetopause, waves in the dayside magnetosheath are controlled by the magnetosphere obstacle and the resultant pileup of the magnetic field: it is not certain that this process operates in the magnetosheath flanks. We conclude that the density fluctuations are probably weaker in the flanks than in the dayside magnetosheath, at least downstream of shocks with the same $\theta_{B N}$.

\subsection{The scattering coefficient as a function of the compression of the magnetosheath?}

We have seen that our two case-studies do not allow us to determine whether a more compressed magnetosheath scatters the radio waves more efficiently. Rezeau et al. (1992) found that the level of the magnetic fluctuations, close to the magnetopause, was higher when the magnetopause moves Earthwards (compression), and lower when it moves outwards (expansion). We can compare the values of $b\left(2 f_{p}\right)$ close to the bow shock, on the two days with shocks moving inwards and outwards. On day $247 / 80$ (Fig. 1) $b\left(2 f_{p}\right.$ ) is larger at 18:07 and 18:58 UT (Earthward shocks) than at 18:14 (outward shock). But on day 188/81 (Fig. 5) $b\left(2 f_{p}\right)$ is larger for the outward-moving shock at 04:10 UT: the level of the density fluctuations is not systematically higher when the bow shock moves towards the Earth.

The fluctuation level in the bow shock probably depends on the parameters given in Table $1, \beta_{u}, M_{A N}$ and $\theta_{B N}$ (Formisano and Hedgecock, 1973; Winterhalter and Kivelson, 1988; Farris et al., 1992) as well as on the shock motion and position. The density fluctuations were measured on ISEE 1-2 during several shock crossings. In this work, we have analysed quasi-perpendicular shocks (see Table 1). The scattering coefficient does not seem to be larger downstream of quasi-parallel shocks: on day 278/81 (not shown) for which $\theta_{B N}=10$ to $20^{\circ}$, $\beta_{u} \simeq 2, M_{A N} \simeq 8$, at 11:40 $\mathrm{LT}, b\left(2 f_{p}\right)$ remains smaller than $20 \times 10^{-9} \mathrm{rad}^{2} / \mathrm{m}$ just downstream of the shock. But this is an underestimation of $b\left(2 f_{p}\right)$ because the separation between ISEE 1 and 2 was $480 \mathrm{~km}$ on that day: $P_{N}(v)$ is underestimated at high frequencies when the separation is larger than about $1 / 4$ of the wavelength of the density fluctuation (see Eq. 15). Typical wavelengths are about 1000 to $3000 \mathrm{~km}$ for mirror modes (Fazakerley and Southwood, 1994; Hubert et al., manuscript in preparation 1997), and $1000 \mathrm{~km}$ for Alfvénic waves (Lacombe et al., 1995). We have therefore not used the density data when the separation was larger than $250 \mathrm{~km}$, and we are not able to check how $b\left(2 f_{p}\right)$ depends on $\beta, M_{A N}$ and $\theta_{B N}$. It would be interesting to check how the level and the scales of the density fluctuations depend on the shock parameters (and the helium content), a work which has been undertaken only for the magnetic fluctuations (see Russell and Farris, 1995).

\subsection{Thickness of the regions of enhanced scattering}

Just downstream of the bow shock, a region of enhanced scattering is observed during about $10 \mathrm{~min}$ (Figs. 1, 3 and 5). With the spacecraft velocity $1-2.5 \mathrm{~km} / \mathrm{s}$, the corresponding thickness is $600-1500 \mathrm{~km}$. But the multiple shock crossings (Figs. 1 and 5) give evidence of back and forth shock motions, with a variable velocity with respect to the spacecraft: this shock velocity can be ten times larger than the spacecraft velocity. The thickness of the region of enhanced scattering is thus probably around $0.1-1$ Earth radius downstream of quasi-perpendicular shocks. It is 10-100 times larger than the downstream proton inertial lengths given in Table 1 . The enhanced scattering just downstream of quasi-perpendicular shocks is probably due to waves generated at the bow shock, by unstable proton distribution functions, which are damped downstream where the distribution functions are marginally stable.

\subsection{Scattering in the proton foreshock?}

We have not calculated the scattering coefficient in the solar wind, upstream of the bow shock because the density data are not good: the $683-\mathrm{kHz}$ wave which allows the density measure, in the propagation experiment, is generally perturbed by the shock ramp (this perturbation can be due to surface waves at the shock front); the $683-\mathrm{kHz}$ wave is also perturbed by the electrostatic waves close to $f_{p}$ typical of the electron foreshock. Upstream of quasi-perpendicular shocks, the scattering coefficient is probably close to the values deduced from the angular radius of the $2 f_{p}$ sources, $b\left(2 f_{p}\right)=2$ to $4 \times 10^{-10} \mathrm{rad}^{2} / \mathrm{m}$ (Lacombe et al., 1988) upstream of the electron foreshock. Upstream of quasiparallel shocks, in the dayside proton foreshock $\left(X_{G S E}>0\right)$, Spangler et al. (1988) found that the relative fluctuations $\sigma_{N} / N$ reached $14 \%$ and $17 \%$ (with fluctuation periods in the range $30 \mathrm{~min}$ to $24 \mathrm{~s}$ ). These percentages are slightly larger than those found just downstream of the shock front $\Delta N / N \leq 10 \%$ to $14 \%$ (see Sect. 4). Shall we conclude that the angular scattering coefficient is larger in the proton foreshock than just downstream of the shock? Even if $\Delta N / N$, the scale $a$ of the density fluctuations and thus $b\left(2 f_{p}\right)$ were the same in the proton foreshock and downstream of the shock, Eq. 11 implies that the scattering coefficient at a frequency $f$ would be smaller in the foreshock where the plasma frequency $f_{p}$ is smaller.

However, the angular scattering in the proton foreshock can play an important part if the proton foreshock region, through which ISEE 3 sees the AKR sources, is much thicker than 0.1 to $1 R_{E}$, or even thicker than the magnetosheath itself. Indeed, a larger propagation distance through a region with smaller $b\left(2 f_{p}\right)$ may lead to larger scattering than a smaller 
propagation distance through a region with larger $b\left(2 f_{p}\right)$. The thickness of the dawn region of the proton foreshock depends on the orientation of the solar wind magnetic field. In a future work, we shall check whether the scattering coefficient, as deduced by Steinberg and Hoang (1993), depends on this orientation.

\subsection{Another method?}

A basic assumption of the present work is that the $a d$ hoc scattering coefficient $b\left(2 f_{p}\right)$ deduced from the ISEE 3 observations and from the ray tracing by Steinberg and Hoang (1993) is meaningful. An argument in favour of this meaning is that the same ad hoc value is able to explain both the angular radius of the AKR source, which can reach $30^{\circ}$, and the direction of the apparent source, which can be more than $60^{\circ}$ tailward of the Earth's direction. But is it possible to derive the turbulent properties of the magnetosheath from the ISEE 3 observations with a method other than ray tracing with the ad hoc $b\left(2 f_{p}\right)$ ? At radio frequencies much higher than $30 \mathrm{kHz}$, the scattering coefficient and spectrum of the density fluctuations in a thin turbulent screen can be derived by a very different method: a radio receiver and antenna with a high angular resolution gives the angular spectrum of a source observed through the turbulent screen (see Bastian, 1994, and references therein); this angular spectrum allows the determination of the screen density spectrum under two main assumptions: i) the density spectrum has a self-similar shape through the screen, ii) the wave propagation through the screen obeys a parabolic wave equation, which does not take into account refraction and backscattering with respect to the main path direction (Cairns, manuscript in preparation, 1997). However, this powerful method cannot be used across the magnetosheath for two reasons: i) there is still no radio interferometer in the solar wind (Spangler and Armstrong, 1990) and the angular resolution of ISEE 3 is much too low; ii) the parabolic wave approximation is not valid because refraction and backscattering are important at frequencies close to the plasma frequencies, and because the density spectra in the magnetosheath are not really self similar. Conversely, the method of Steinberg and Hoang (1993) takes into account refraction and backscattering; it could also take into account the fact that $b\left(2 f_{p}\right)$ is found to vary through the magnetosheath.

\section{Conclusion}

ISEE 1-2 are the only spacecraft to have measured in situ the electron density fluctuations up to $v=1 \mathrm{~Hz}$ or more. Frequency spectra of these fluctuations have been obtained during two complete crossings of the dayside magnetosheath, downstream of the quasi-perpendicular bow shock. The angular scattering coefficient $b(f)$ of a radio wave with a frequency $f$ can then be calculated by integrating the inverse of the scale length of the density fluctuations over the spectrum of these fluctuations, from $v_{\min }=0.0156 \mathrm{~Hz}$ to $v_{\max }=1 \mathrm{~Hz}$.

The local scattering coefficient has thus been calculated with a realistic spectrum of the density fluctuations, at high enough frequencies. The relative density fluctuation $\Delta N / N$ is always smaller than $15 \%$. At twice the electron plasma frequency, the scattering coefficient in the major part of the dayside magnetosheath is found to be $b\left(2 f_{p}\right) \simeq 0.5-4 \times 10^{-9} \mathrm{rad}^{2} / \mathrm{m}$, taking into account the angular deviation in two orthogonal planes. The relative uncertainty on $b\left(2 f_{p}\right)$ is $(\Delta b) / b=1-2$. Close to the bow shock (and also close to the magnetopause on one day), $b\left(2 f_{p}\right)$ reaches $10-30 \times 10^{-9} \mathrm{rad}^{2} / \mathrm{m}: b\left(2 f_{p}\right)$ is multiplied by about 10 in a sheet with a thickness of $0.1-$ $1 R_{E}$. These values of $b\left(2 f_{p}\right)$ are close enough to the average scattering coefficient $4 \times 10^{-9} \mathrm{rad}^{2} / \mathrm{m}$ deduced from several observations of the AKR propagating through the dawn flank of the magnetosheath and from the ray tracing (Steinberg and Hoang, 1993). This agreement implies that the density fluctuations which produce the largest part of the scattering have been observed by ISEE 1 and ISEE 2 when the density was averaged over an inter-spacecraft separation distance of $200 \mathrm{~km}$ (or less) with a sampling time of $0.25 \mathrm{~s}$ (or less). This agreement also implies that, if there is another scattering process of radio waves in the Earth's magnetosheath (for instance by ripples on the shock front), this other process is not significantly more efficient than the scattering by refractive index fluctuations.

A precise knowledge of the density fluctuations in the magnetosheath is necessary to understand the propagation of low-frequency radio waves. Conversely, observations of low-frequency terrestrial sources through the magnetosheath can give information on the overall structure and properties of the magnetosheath and the bow shock.

Acknowledgements. We are grateful to I. H. Cairns, Y. Leblanc and G. A. Dulk for useful suggestions on the original manuscript. We thank the referee for interesting and stimulating comments. We thank C. T. Russell for the ISEE 1-2 magnetometer data which have been used for the determination of the shock parameters.

Topical Editor K.-H. Glaßmeier thanks S. R. Spangler for his help in evaluating this paper.

\section{References}

Alexander, J. K., M. L. Kaiser, and P. Rodriguez, Scattering of terrestrial kilometric radiation at very high altitudes, $J$. Geophys. Res., 84, 2619-2629, 1979.

Bastian, T. S., Angular scattering of solar radio emission by coronal turbulence, Astrophys. J., 426, 774-781, 1994.

Cairns, I. H., Radio wave scattering in the outer heliosphere: preliminary calculations, Geophys. Res. Lett., 22, 3433-3436, 1995.

Celnikier, L. M., C. C. Harvey, R. Jegou, M. Kemp, and P. Moricet, A determination of the electron density fluctuation spectrum in the solar wind, using the ISEE propagation experiment, Astron. Astrophys., 126, 293-298, 1983.

Celnikier, L. M., L. Muschietti, and M. V. Goldman, Aspects of interplanetary turbulence, Astron. Astrophys., 181, 138-154, 1987. 
Couzens, D. A., and J. H. King, Interplanetary Medium Data Book Supplement 3a, 1977-1985, NSSDC/ WDCA, 86-04a, 1986.

Fairfield, D. H., and N. F. Ness, Magnetic field fluctuations in the Earth's magnetosheath, J. Geophys. Res., 75, 6050-6060, 1970.

Farris, M. H., C. T. Russell, M. F. Thomsen, and J. T. Gosling, ISEE 1 and 2 observations of the high beta shock, J. Geophys. Res., 97, 19121-19127, 1992.

Fazakerley, A. N., and D. J. Southwood, Mirror instability in the magnetosheath, Adv. Space Res., 14(7), 65-68, 1994.

Filbert, P. C., and P. J. Kellogg, Electrostatic noise at the plasma frequency beyond the Earth's bow shock, J. Geophys. Res., 84, 1369-1381, 1979.

Formisano, V., and P. C. Hedgecock, Solar wind interaction with the Earth's magnetic field, 3. On the Earth's bow shock structure, J. Geophys. Res., 78, 3745-3760, 1973.

Harvey, C. C., J. Etcheto, Y. de Javel, R. Manning, and M. Petit, The ISEE electron density experiment, IEEE Trans. Geosci. Electron., GE-16, 231-238, 1978.

Hollweg, J. V., Angular broadening of radio sources by solar wind turbulence, J. Geophys. Res., 75, 3715-3727, 1970.

Hubert, D., Nature and origin of wave modes in the dayside Earth magnetosheath, Adv. Space Res., 14(7), 55-64, 1994.

Huddleston, D. E., R. Woo, and M. Neugebauer, Density fluctuations in different types of solar wind flow at $1 \mathrm{AU}$ and comparison with results from Doppler scintillation measurements near the Sun, J. Geophys. Res., 100, 19951-19956, 1995.

Lacombe, C., C. C. Harvey, S. Hoang, A. Mangeney, J.-L. Steinberg, and D. Burgess, ISEE observations of radiation at twice the solar wind plasma frequency, Ann. Geophysicae, 6, 113-128, 1988.

Lacombe, C., F. G. E. Pantellini, D. Hubert, C. C. Harvey, A. Mangeney, G. Belmont, and C. T. Russell, Mirror and Alfvénic waves observed by ISEE 1-2 during crossings of the Earth's bow shock, Ann. Geophysicae, 10, 772-784, 1992.

Lacombe, C., G. Belmont, D. Hubert, C. C. Harvey, A. Mangeney, C. T. Russell, J. T. Gosling, and S. A. Fuselier, Density and magnetic field fluctuations observed by ISEE 1-2 in the quiet magnetosheath, Ann. Geophysicae, 13, 343-357, 1995.

Moustaizis, S., D. Hubert, A. Mangeney, C. C. Harvey, C. Perche, and C. T. Russell, Magnetohydrodynamic turbulence in the Earth magnetosheath, Ann. Geophysicae, 4A, 355-362, 1986.

Peredo, M., J. A. Slavin, E. Mazur, and S. A. Curtis, Threedimensional position and shape of the bow shock and their variation with Alfvénic, sonic and magnetosonic Mach numbers and interplanetary magnetic field orientation, J. Geophys. Res., 100, 7907-7916, 1995.

Press, W. H., S. A. Teukolsky, W. T. Vetterling, and B. P. Flannery, Numerical recipes in FORTRAN, 2nd Edition, Cambridge University Press, Cambridge, 1992.
Rezeau, L., A. Roux, and C. T., Russell, Can ULF fluctuations observed at the magnetopause play a role in anomalous diffusion? Proc. 26th ESLAB Symp., Killarney, 127-131, 1992.

Russell, C. T., and M. H. Farris, Ultra low frequency waves at the Earth's bow shock, Adv. Space Res., 15(8/9), 285-296, 1995.

Sibeck, D. G., R. E. Lopez, and E. C. Roelof, Solar wind control of the magnetopause shape, location and motion, J. Geophys. Res., 96, 5489-5495, 1991.

Spangler, S. R., and J. W. Armstrong, Low-frequency angular broadening and diffuse interstellar plasma turbulence, in Low frequency astrophysics from space, Eds. N.E. Kassim and K.W. Weiler, Springer- Verlag, Berlin, Heidelberg, New York, 155164, 1990.

Spangler, S. R., S. A. Fuselier, A. L. Fey, and G. Anderson, An observational study of MHD wave-induced density fluctuations upstream of the Earth's bow shock, J. Geophys. Res., 93, 845$857,1988$.

Spreiter, J. R., and S. S. Stahara, Magnetohydrodynamic and gasdynamic theories for planetary bow waves, in Collisionless shocks in the heliosphere: reviews of current research, Eds. B.T. Tsurutani and R.G. Stone, Geophysical Monograph 35, AGU, 85-107, 1985.

Steinberg, J.-L., and S. Hoang, Magnetosheath density fluctuations from a simulation of auroral kilometric radiation radio propagation, J. Geophys. Res., 98, 13467-13475, 1993.

Steinberg, J.-L., and C. Lacombe, An empirical model of the plasma density distribution in the distant Earth magnetosheath, Geophys. Res. Lett., 19, 2285-2288, 1992.

Steinberg, J.-L., M. Aubier- Giraud, Y. Leblanc, and A. Boischot, Coronal scattering, absorption and refraction of solar radio bursts, Astron. Astrophys., 10, 362-376, 1971.

Steinberg, J.-L., S. Hoang, and G. A. Dulk, Evidence of scattering effects on the size of interplanetary Type III radio bursts, Astron. Astrophys., 150, 205-216, 1985.

Steinberg, J.-L., S. Hoang, and C. Lacombe, Propagation of terrestrial kilometric radiation through the magnetosheath: ISEE-3 observations, Ann. Geophysicae, 7, 151-160, 1989.

Steinberg, J.-L., S. Hoang, J.-M. Bosqued, Isotropic terestrial kilometric radiation: a new component of the Earth's radio emission, Ann. Geophysicae, 8, 671-686, 1990.

Winterhalter, D., and M. G. Kivelson, Observations of the Earth's bow shock under high Mach number/high plasma beta solar wind conditions, Geophys. Res. Lett., 15, 1161-1164, 1988.

Woo, R., A synoptic study of Doppler scintillation transients in the solar wind, J. Geophys. Res., 93, 3919-3926, 1988. 